\title{
Heparanase modulates Shh and Wnt3a signaling in human medulloblastoma cells
}

\author{
LON D. RIDGWAY, MICHAEL D. WETZEL and DARIO MARCHETTI \\ Department of Pathology and Immunology, Baylor College of Medicine, Houston, TX 77030, USA
}

Received November 8, 2010; Accepted December 3, 2010

DOI: $10.3892 / \mathrm{etm} .2010 .189$

\begin{abstract}
The pathogenesis of medulloblastoma (MB), the most common and aggressive brain tumor in children, is poorly understood. MB tumors respond to factors secreted by cerebellar Purkinje neurons, such as Sonic hedgehog (Shh) and Wingless-type MMTV integration site family member 3a (Wnt3a). Understanding the modulation of Shh/Wnt signaling is critical for the development of new MB treatments. Shh and Wnt3a induce MB cell proliferation and bind heparan sulfate glycosaminoglycan chains (HS-GAGs). HS-GAGs are components of syndecans, cell surface HS proteoglycans (HSPGs) which act as co-receptors for extracellular matrixbased ligands, and are targets of heparanase (HPSE). We hypothesized that extracellular HPSE activity modulates MB intracellular signaling of Shh/Wnt3a, involving syndecan-1 and -4 carboxy terminal-associated proteins and downstream targets. We compared the regulation of Shh/Wnt3a signaling subsequent to treatment with exogenous human active HPSE
\end{abstract}

Correspondence to: Professor Dario Marchetti, Department of Pathology and Immunology, Baylor College of Medicine, BCM Taub Bldg., Suite T240A, Mail stop 315, One Baylor Plaza, Houston, TX 77030, USA

E-mail: marchett@bcm.edu

Abbreviations: CT, carboxy terminal; DEPC, diethylpyrocarbonate; ECM, extracellular matrix; FBS, fetal bovine serum; GAG, glycosaminoglycan; GEF-H1, guanine nucleotide exchange factor-H1; GPC, glypican; GPI, glycosylphosphotidylinositol; GST, glutathioneS-transferase; GSTPD, GST pulldown; $\mathrm{H}_{2} \mathrm{O}$, double-distilled water; HEPES, 4-(2-hydroxyethyl)-piperazine-1-ethanesulfonic acid, $\mathrm{N}$-(2-hydroxyethyl)piperazine-N'-(2-ethanesulfonic acid); rhHPSE, recombinant human active heparanase (GS3); HS, heparan sulfate; HSPG, heparan sulfate proteoglycan; IPTG, isoprythiogalactoside; MALDI-TOF MS/MS, matrix-assisted laser desorption ionization time of flight tandem mass spectrometry; $\mathrm{MB}$, medulloblastoma; MEM, minimal essential media; Shh, recombinant human Sonic hedgehog; Wnt3a, recombinant human Wingless-type MMTV integration site family member 3a; SDC, syndecan; SDS-PAGE, sodium dodecyl sulfate-polyacrylamide gel electrophoresis; Wnt, Wingless-type MMTV integration site

Key words: heparanase, medulloblastoma, Sonic hedgehog, Wingless-type MMTV integration site family member 3a, guanine nucleotide exchange factor-H1, GTPase in $\mathrm{MB}$ lines possessing increased invasive abilities. We identified guanine nucleotide exchange factor-H1 (GEF-H1), a small GTPase guanine nucleotide exchange factor, as a new component of a syndecan signaling complex. Secondly, we demonstrated that HPSE modulated Shh/Wnt3-dependent expression and the intracellular distribution of GEF-H1, $\beta$-catenin and N-Myc. Thirdly, HPSE modulated Shh/Wnt3adependent gene expression of heparan sulfate proteoglycan (HSPG) and Gli transcription factors. Fourthly, pre-treatment with HPSE, alone or prior to Shh/Wnt3a exposure, altered small GTPase (Rac1/RhoA) activities differentially and promoted RhoA activation. Finally, the differential regulation of Rac1/RhoA activities by HPSE affected MB cell proliferation and invasion. Our results indicate that the HPSE/HSPG axis is implicated in critical MB cell signaling pathways with potential relevance for MB treatment.

\section{Introduction}

Medulloblastoma (MB) is the most common invasive brain tumor of childhood. It arises in the cerebellum, generally occurs during embryogenesis and is characterized by a massive expansion of cells from the external granular layer. These events are thought to originate in response to growth factor secretion from Purkinje neurons, such as Sonic hedgehog (Shh) and Wingless-type MMTV integration site family member 3a (Wnt3a) (1,2). An insidious feature of MB tumors is their metastatic propensity as evident from MB cell intravasation through the fourth ventricle into the cerebral spinal fluid, resulting in dissemination throughout the spinal cord $(1,3,4)$. Despite advances in therapy, a significant number of patients succumb to the disease, and survivors often suffer permanent neurocognitive deficiencies (3-5).

Multiple signaling pathways have been associated with MB onset and growth, including Shh and Wnt. Shh is a secreted protein that binds to the transmembrane receptor Ptch1. Upon Shh binding, Ptch1 inhibition of Smoothened is relieved and Shh signal is transduced to the Gli family of transcription factors $(6,7)$.

A key feature of both Shh and Wnt3a is their highaffinity binding to heparan sulfate glycosaminoglycan chains (HS-GAGs) (8). For example, treatment of cerebellar sections with bacterial heparinases abrogates Shh mitogenicity (9). HS-GAGs are components of HS proteoglycans (HSPGs), ubiquitous macro-molecules located in the extracellular matrix 
and on the cell surface, consisting of a core protein and covalently attached HS-GAGs (10-14). Cell surface HSPGs, as members of the syndecan and glypican families, regulate cross-talk between tumor and host cells by controlling the location and activity of growth factors, acting as co-receptors. Their unique functions place HSPGs at the center of cell signaling integration (15).

HSPG expression characteristics have been linked to tumor cell metastasis (11-14). Notably, HSPG are targets of heparanase (HPSE), the dominant mammalian endoglycosidase (endo- $\beta$-D-glucuronidase) whose activity has been proven to be implicated in the angiogenic, tumorigenic and metastatic abilities of tumor cells $(16,17)$. HPSE cleaves HS-GAGs at specific intrachain sites resulting in fragments (10-20 sugar subunits) which are biologically active, i.e., able to bind growth and angiogenic factors. Although Shh roles in $\mathrm{MB}$ have been demonstrated $(2,18,19)$, mechanistic links between Shh deregulation and HPSE have yet to be investigated. Similarly, Wnt pathway activation involves the interaction of a Wnt ligand with Frizzled receptors, which leads to inhibition of the $\beta$-catenin regulatory complex. Modulation of Wnt signaling by HPSE has not been addressed, nor the mechanisms or functional links between Wnt activation and MB (20).

The invasive cell phenotype requires cytoskeletal dynamics driven by the small GTPases, Rac and Rho $(21,22)$. As tumor cells invade the surrounding tissue, they establish, abolish and/or relocate transient focal adhesions $(22,23)$. Focal adhesion dynamics require Rac and Rho activities (21), and recent evidence supports roles of syndecan (SDC) cell surface HSPGs (24).

We hypothesized that SDC downstream signaling events are crucial for driving MB cell proliferation and invasiveness, and that HPSE modulates HSPG-associated Shh/Wnt3a actions. To this end, we utilized human MB lines (D721 and D283) that we had previously characterized, establishing gradients of cell invasiveness and HPSE functionality $(4,25)$. We identified GEF-H1 as a novel SDC-associated signal transduction protein in human MB cells. Of note, GEF-H1 is a Rho guanine nucleotide exchange factor with microtubule binding ability which also binds Racl as an inhibitor (26). We investigated GEF-H1, $\beta$-catenin and N-Myc protein expression and subcellular localization, in addition to cell surface HSPG and Gli 1-3 gene expression, by pre-treating MB cells with human active HPSE, followed by Shh or Wnt3a exposure. In this study, we demonstrate the roles of HSPG linking Shh/Wnt3a signaling to GEF-H1, Rac1/RhoA activities and MB cell proliferative and invasive events. Furthermore, we provide evidence that HPSE modifies MB cell responses to HS-binding Shh and Wnt3a.

\section{Materials and methods}

Cell culture and treatments. Human MB cell lines of low/high invasive abilities (D283 and D721) (4) were kindly provided at low-passage by Dr Darrell Bigner (Duke University, Durham, NC, USA) and cultured in improved minimal essential media (IMEM) with Richter's $\mathrm{Zn}^{2+}$ Option (Richter's modification), $2 \mathrm{mg} / \mathrm{l} \mathrm{L}$-glutamine, $2 \mathrm{mg} / \mathrm{l} \mathrm{L}$-proline, $50 \mu \mathrm{g} / \mathrm{ml}$ gentamicin sulfate (Gibco/Invitrogen, Carlsbad, CA, USA) supplemented with $20 \%$ fetal bovine serum (FBS), $100 \mathrm{U} / \mathrm{ml}$ penicillin and $100 \mu \mathrm{g} / \mathrm{ml}$ streptomycin (normal growth medium). The cultures were maintained at $37^{\circ} \mathrm{C}$ in $5 \% \quad \mathrm{CO}_{2}$. Due to the inherent difficulty in growing $\mathrm{MB}$ cells, special care was taken to grow cultures in flasks maintained in a canted position and at high cell density. HPSE treatments were for $12 \mathrm{~h}(200 \mathrm{ng} /$ $\mathrm{ml}$ ) using recombinant human active heparanase (rhHPSE) in IMEM with Richter's $\mathrm{Zn}^{2+}$ Option (Richter's modification), $2 \mathrm{mg} / \mathrm{l} \mathrm{L}$-glutamine, $2 \mathrm{mg} / \mathrm{l} \mathrm{L}$-proline, $50 \mu \mathrm{g} / \mathrm{ml}$ gentamicin sulfate supplemented with $10 \mathrm{mM}$ HEPES (pH 6.5), 5\% FBS, $100 \mathrm{U} / \mathrm{ml}$ penicillin and $100 \mu \mathrm{g} / \mathrm{ml}$ streptomycin. The cells were periodically tested for Mycoplasma negativity, and only low passage cells were used in the experiments.

Heparanase, HepIII, Shh and Wnt treatments. Preparations of highly active rhHPSE were kindly provided by Dr Israel Vlodavsky (The Bruce Rappaport Medical Faculty, Technion, Israel) (27). Heparanase activity was validated by utilizing a heparan sulfate-degrading enzyme assay kit (Takara Mirus, Madison, WI, USA). Following the mock or HPSE treatment, the cells were serum-starved for either 12 or $48 \mathrm{~h}$, as indicated. The growth factor treatments were for $12 \mathrm{~h}$ with either recombinant human Shh (R\&D Systems, Minneapolis, MN, USA) or recombinant human Wnt3a (R\&D Systems) (100 ng/ $\mathrm{ml}$ each). The removal of HS-GAGs of cell surface HSPG was accomplished by digestion with Heparitinase III (HepIII) from Flavobacterium heparinum (Sigma, St. Louis, MO, USA). The final HepIII concentration was $0.05 \mathrm{U} / \mathrm{ml}$ culture medium. The digestions were carried out for $1 \mathrm{~h}$ at $37^{\circ} \mathrm{C}$ in $5 \% \mathrm{CO}_{2}$.

$R T-P C R$. Total RNA was isolated from the MB cells using the RNeasy Plus mini-kit (Qiagen, Valencia, CA, USA) according to the manufacturer's instructions. The RNA yield was determined using a NanoDrop ND1000 spectrophotometer (NanoDrop Products, Wilmington, DE, USA). To ensure a lack of genomic DNA contamination, $1 \mu \mathrm{g}$ of total RNA was digested with DNase I (Invitrogen) prior to first-strand synthesis. Inactivated DNase I digestion $(2 \mu \mathrm{l})$ was used as a template with a first-strand synthesis kit utilizing SuperScript II reverse transcriptase according to the manufacturer's instructions (Invitrogen). The first-strand synthesis reaction was then diluted $1: 1$ with $\mathrm{H}_{2} \mathrm{O}$ to be utilized as a single-strand cDNA template. PCR amplification was performed in 20- $\mu 1$ reactions consisting of 1X AmpliTaq Gold buffer (Applied Biosystems, Foster City, CA, USA), $2 \mathrm{mM} \mathrm{MgCl}_{2}, 300 \mu \mathrm{M}$ dNTP mix, $400 \mathrm{nM}$ primer pair, $2 \mu \mathrm{l}$ single strand cDNA template and 2 units AmpliTaq Gold Taq polymerase (Applied Biosystems). PCR conditions were: $94^{\circ} \mathrm{C}, 2 \mathrm{~min} ; 40$ cycles of $94^{\circ} \mathrm{C}, 20 \mathrm{sec}$; $58^{\circ} \mathrm{C}, 15 \mathrm{sec} ; 72^{\circ} \mathrm{C}, 42 \mathrm{sec} ; 72^{\circ} \mathrm{C}, 30 \mathrm{sec}$. Gene accession numbers and DNA sequences for the oligonucleotide primer pairs utilized were: GAPDH, NM_002046.3, forward TTC CAC CCA TGG CAA ATT CC, reverse TGG CAG GTT TTT CTA GAC GG; HPSE, NM_006665.3, forward CTG GCA ATC TCA AGT CAA CC, reverse TCC TAA CCA GAC CTT CTT GC; SDC1, NM_001006946.1, forward TGA CTC TGA CAA CTT CTC CG, reverse TCT TCT TCA TGC GGT ACA GC; SDC2, NM_002998.3, forward AAC CAG AAA CTG AAC CTC GG, reverse TCT ACA TCC TCA TCA GCT CC; SDC3, NM_014654.3, forward AGT GAG AAC TTC GAG AGA CC, reverse TGT GTG GTC TCT TCT TCT GG; SDC4, NM_002999.2, forward TAG AAG GCC GAT 
ACT TCT CC, reverse TCA CGC GTA GAA CTC ATT GG; GPC1, NM_002081.2, forward AGC CAT GTA TTT CAG GGA CC, reverse GGA TGC ATG TTT GGA AAA GC; GPC2 NM_152742.1, forward CTC GGT ATT CAG TTT TCC GG, reverse TTT CCC CAT AGA AGT CTC GC; GPC3, NM_004484.2, forward GCA AGT ATG TCT CCC TAA GG, reverse CTT GCA GTG ACT TGG AAA CC; GPC4, NM_001448.2, forward CAA GCT GTC TTT GCT TCA CG, reverse AAC ATG GCT TCA CAG TCA CG; GPC5, U66033.1, forward GAC CTG ATC TTC AGG TTT GC, reverse GAA GTG CAG ATA GTC TGT GG; GPC6, NM_005708.2, forward TCC TCG TTT TGA TTG CAC CG, reverse AAG TGG TGC GCA CAA AAT GG; Gli1, NM_005269, forward ACC AAT CAG TAG CTA TGG CG, reverse TAT CAC CTT CCA AGG GTT CC; Gli2, NM_005270.4, forward ACA TCA ACA ACT CCC GAA GC, reverse TGT CCA GAT CTT CCT TGA GG; Gli3, NM_000168.5, forward TCT CCA TGA TCT CAG CAA CC, reverse AGA GTA GGT GAA GCT CAA GG. The PCR reactions were conducted in a Mastercycler ep gradient thermocycler (Eppendorf North America, Westbury, NY, USA).

Cloning. For affinity chromatography experiments, the pGEX-4T-3 vector was used (GE Healthcare, Piscataway, NJ, USA). RT-PCR was performed to amplify cDNA regions encoding the intracellular CT domains. Oligonucleotide primer pairs were designed for insertion into the Bam HI/XhoI (New England Biolabs, Ipswich, MA, USA) restriction sites within the pGEX vector. The gene accession numbers and DNA sequences for the oligonucleotide primer pairs utilized were: SDC1, NM_001006946.1, GST SDCN1 CT forward TTT GGA TCC CGC ATG AAG AAG AAG GAC GA; GST SDCN1 CT reverse TTT CTC GAG TCA GGC ATA GAA TTC CTC CTG; SDC4, NM_002999.2, GST SDCN4 CT forward TTT GGA TCC CGT ATG AAG AAG AAG GAT GAA G; GST SDCN4 CT reverse TTT CTC GAG TCA CGC GTA GAA CTC ATT GGT. The accuracy of these DNA sequences was confirmed by sequencing (SeqWright Inc., Houston, TX, USA).

Mass spectrometry. Protein bands excised from the SDS-PAGE were identified by matrix-assisted laser desorption ionization time of flight tandem mass spectrometry (MALDI-TOF MS/ MS) from the proteomic core facility at Baylor College of Medicine. Briefly, unique bands were excised from GSTPD and identified binding proteins using MALDI-TOF MS/MS. Protein digests were then spotted on a MALDI target plate, and the analyses were performed using an ABI/SCIEX 4700 Proteomics Analyzer and TOF/TOF mass spectrometer. Detected mono-isotopic peptide masses were then analyzed by MS-Fit (Protein Prospector), and proteins were identified using peptide mass finger-printing.

Cell lysates. RIPA buffer (Sigma) containing $150 \mathrm{mM} \mathrm{NaCl}$, 1\% IGEPAL CA-630 (Sigma), $0.5 \%$ sodium deoxycholate $0.1 \%$ SDS, $50 \mathrm{mM}$ Tris, $\mathrm{pH}$ 8.0, supplemented with the protease inhibitors $1 \mathrm{mM}$ PMSF (Sigma) and Complete-mini (Roche, Mannheim, Germany) was used to generate whole cell lysates. Nuclear and cytoplasmic lysates were generated using the NE-PER kit (Thermo, Waltham, MA, USA) according to the manufacturer's instructions.
GST pulldowns. This procedure was performed as previously described (28). Briefly, GST-SDC1 or GST-SDC4 fusion constructs were transformed into the E. coli strain BL21 (Novagen, Madison, WI, USA). Expression was induced by the addition of IPTG to LB culture media. Induced fusion proteins from the bacteria were purified by lysing the cells using B-PER reagent (Thermo) and passing the cell lysates over columns containing glutathione beads (Thermo). GST fusion proteins were eluted from the columns with reduced glutathione. The purified GST fusion proteins $(30 \mu \mathrm{g})$ were bound to glutathione bead columns, and $500 \mu \mathrm{g}$ of whole cell lysates was passed over the columns. The columns were then washed three times, and the bound proteins were eluted with $2 \mathrm{X}$ Laemmli buffer with subsequent heating for $5 \mathrm{~min}$ at $95^{\circ} \mathrm{C}$. Eluted proteins were detected by Coomassie blue. Bands of interest were excised from the gels and identified by mass spectrometry.

GTPase activity assays. Racl and RhoA small GTPase activity assays were performed using respective GLISA kits (BK124, BK125) according to the manufacturer's instructions (Cytoskeleton, Denver, CO, USA). Briefly, after determining the cell number to cell lysis buffer volume ratio that would reproducibly result in protein concentrations of $0.5 \mu \mathrm{g} / \mu \mathrm{l}$, the experimental treatments were performed. Cell lysates were snap-frozen in liquid nitrogen to preserve GTP-bound GTPase (Rac1 or RhoA). GTP bound Rac1 GTPase was bound by Rac1GTP-binding protein-linked wells of a 96-well plate (Corning). Bound active Racl was detected with a Rac1-specific antibody. The degree of Rac1 activation was determined by comparison to corresponding values obtained from lysates derived from untreated serum-starved cells. GTP-bound RhoA GTPase activity was similarly assayed (GLISA kits; Cytoskeleton). The experiments were replicated, data were analyzed and means/standard deviations were calculated.

Western blotting. Cell lysates were quantified by standard spectrophotometric methods using either Protein Reagent (Bio-Rad, Hercules, CA, USA) or BCA Reagent (Thermo). Aliquots (20 $\mu \mathrm{g}$ of protein) of quantified cell lysates were resolved by 10-12\% SDS-PAGE, transferred onto polyvinylidene difluoride (PVDF) membranes (Bio-Rad) in Tris-buffered saline (TBST) containing 5\% non-fat dry milk and $0.05 \%$ Tween-20 (Sigma). Hybridizations were performed for $16 \mathrm{~h}$ at $4^{\circ} \mathrm{C}$. The antibodies used were: SDC1 (clone B-A38, 854.070.000; Cell Sciences, Canton, MA, USA), SDC4 (ab24511; Abcam, Cambridge, MA, USA), GEF-H1 (55B6; Cell Signaling), N-Myc (9405; Cell Signaling), $\beta$-actin (clone AC-15, sc-69879; Santa Cruz Biotechnology), $\beta$-catenin (9581; Cell Signaling) and Fibrillarin (clone 38-F3, sc-56676; Santa Cruz Biotechnology). Hybridized primary antibodies were detected with ECL reagents (Thermo) and HRP-conjugated secondary goat anti-rabbit (sc-2030) or goat anti-mouse (sc-2031; Santa Cruz Biotechnology) antibodies. Stripping of blots was performed with Restore reagent (21059) (Thermo).

Cell proliferation assays. Cell proliferation was determined by AlamarBlue staining using the manufacturer's protocol (Sigma). Briefly, following treatment conditions, cells $\left(3.0 \times 10^{6}\right)$ were plated in 96-well plates containing growth media and 
$10 \mu \mathrm{l}$ AlamarBlue, and subsequently cultured for $4 \mathrm{~h}$ at $37^{\circ} \mathrm{C}$. Proliferation was determined by measuring OD readings at 570 and $600 \mathrm{~nm}$ using a SpectraMax Plus384 spectrophotometer (Molecular Devices, Sunnyvale, CA, USA). Data were then analyzed for statistical significance. All assays were performed in triplicate.

Invasion assays. Transwell membrane inserts (Corning) were coated with Matrigel ${ }^{\mathrm{TM}}$ (BD Biosciences) at a 1:30 dilution in serum-free media overnight $(16 \mathrm{~h})$ before use. The inserts were washed with serum-free medium, and $1.5 \times 10^{5}$ cells/ insert in IMEM + $0.1 \%$ BSA were placed on the top chambers. The bottom chambers contained IMEM medium with $20 \%$ FBS and N-formyl-Met-Leu-Phe $(5 \mu \mathrm{M})$ as chemoattractant (Sigma). The assays were run for $12 \mathrm{~h}$ and then the inserts were removed, stained with $0.5 \%$ crystal violet solution (400 $\mu$ l) (Sigma) for $10 \mathrm{~min}$, washed and extracted with $200 \mu \mathrm{l}$ of extraction solution (Cell Biolabs, San Diego, CA, USA) for $10 \mathrm{~min}$. The OD values were read at $560 \mathrm{~nm}$ using $100 \mu 1$ of extracted solution and statistically analyzed.

Statistics. Data are represented as the mean \pm standard deviation. Significance values were obtained by the Student's paired t-test $(\mathrm{P}<0.05 ; \mathrm{P}<0.01)$. $\mathrm{P}$-values of $<0.01$ were considered highly significant.

\section{Results}

Differential cell surface HSPG and GEF-H1 expression in medulloblastoma. In previous studies, we demonstrated that heparanase expression of specified MB cell lines correlates with their invasive phenotype (25). We considered that SDCs, particularly SDC1/4, in addition to bearing HS-GAGs as substrates for HPSE enzymatic activity, may have intracellular CT signaling capabilities that have yet to be characterized. We hypothesized that HPSE modulates downstream signaling events that are dependent upon HS/HS-binding growth factor interactions. To determine the overall cell surface HSPG expression status in these $\mathrm{MB}$ cell lines, semi-quantitative RT-PCR was performed and gene expression levels for members of the SDC and glypican families were determined (12). We focused on SDC1/4, considering their established roles in cell invasion, cell adhesion and cytoskeletal rearrangement $(13,29)$. Furthermore, SDC1 has been reported to affect Wnt responsiveness in other model systems (30), and SDC4 is a fundamental component of focal adhesions (31). Unique cell surface HSPG gene expression patterns were observed for each MB cell line analyzed (Fig. 1A). Notably, SDC1/4 gene profiling from D283 and D721 cells was detected under normal growth conditions (Fig. 1A).

Next, to identify SDC intracellular CT binding signal transduction components, GST fusion proteins were constructed containing the intracellular CT domains from human SDC1/4 and performed GST pulldown assays using cell lysates from D283 and D721 MB cell lines. Proteins from the GST pulldown eluates were then separated by SDS-PAGE. A single Coomassie blue-stained protein band of $110 \mathrm{kDa}$ was eluted preferentially from the D283 cell lysates compared to the D721 eluted proteins (Fig. 1B). This band was excised, subjected to trypsin digestion and the resulting peptides were identified by
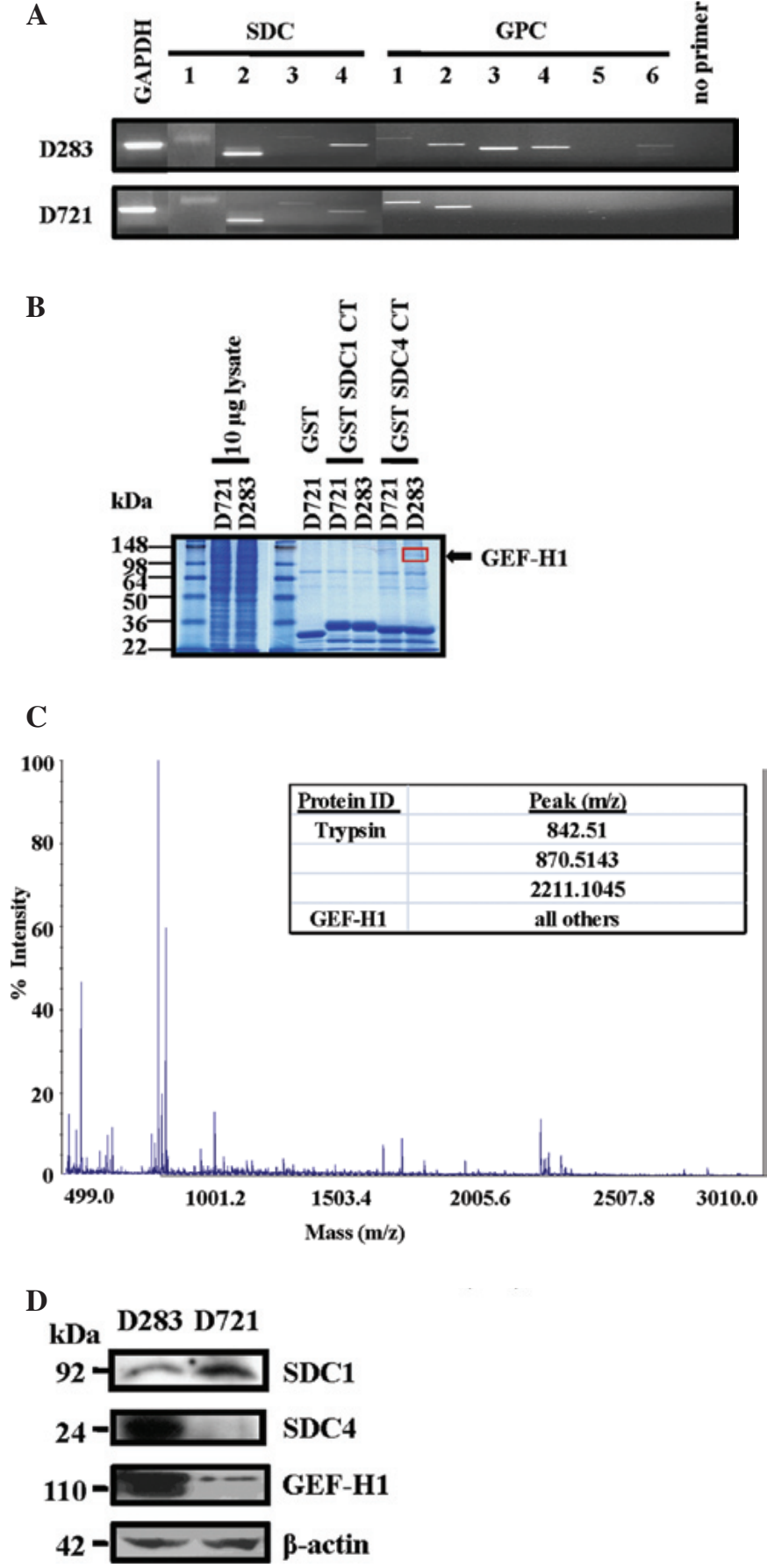

Figure 1. Medulloblastoma cell lines express cell surface HSPGs and GEF-H1 is a component of a SDC CT-binding complex. (A) Semi-quantitative RT-PCR analysis of D283 and D721 cells. GAPDH was used as a positive control. Mastermix with the primer pair was used as a negative control and a test for contamination. PCR product sizes: GAPDH, $611 \mathrm{bp}$; SDC1, $725 \mathrm{bp}$; SDC2, 548 bp; SDC3, 824 bp; SDC4 500 bp; GPC1, 849 bp; GPC2, 602 bp; GPC3, 488 bp; GPC4, 517 bp; GPC5, 569 bp; GPC6, 573 bp. (B) SDS-PAGE of GST pulldown eluates. The arrow indicates the endogenously-expressed SDC1/4 CT binding protein that was excised from a Coomassie blue-stained gel of GST pulldown eluates. (C) GEF-H1 is identified by MALDI-TOF $\mathrm{MS} / \mathrm{MS}$ as a SDC4 CT-associated protein from the D283 cell lysate, per B. (D) Representative Western blotting detecting endogenous SDC1 (core protein), SDC4 (core protein) and GEF-H1 in poorly (D283) and highly (D721) invasive MB cells. $\beta$-actin was used as a control for equal loading.

MALDI-TOF tandem mass spectrometry. This unique band was identified as human GEF-H1 (Fig. 1C). Furthermore, $\mathrm{SDC} 1 / 4$ and GEF-H1 protein expression was visualized in 
A

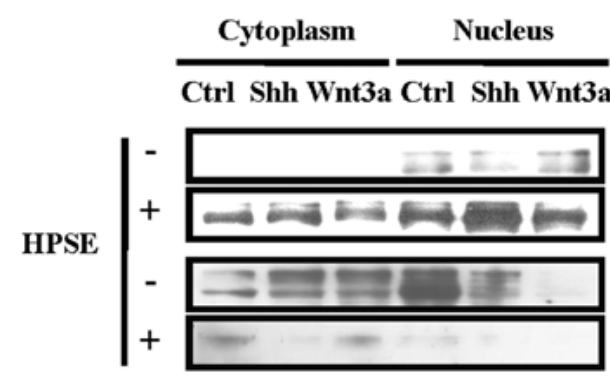

C

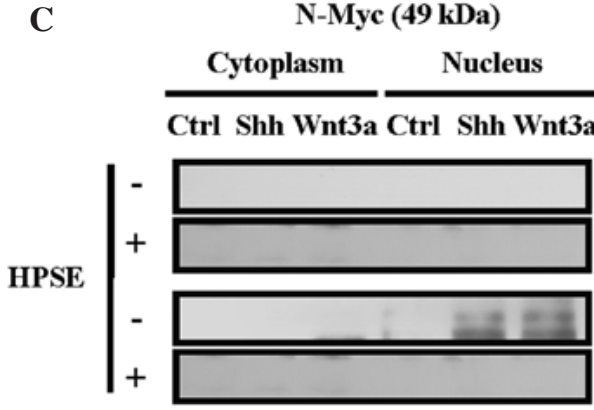

B B-Catenin (94 kDa)

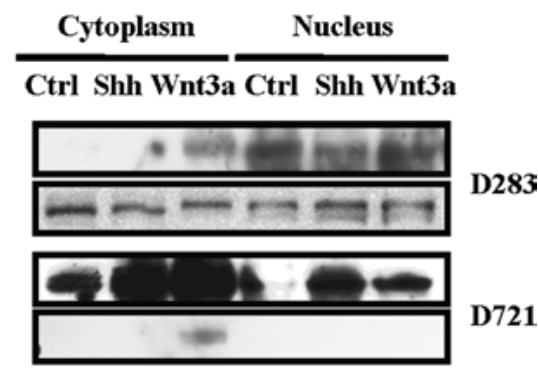

D Fibrillarin (36 kDa)

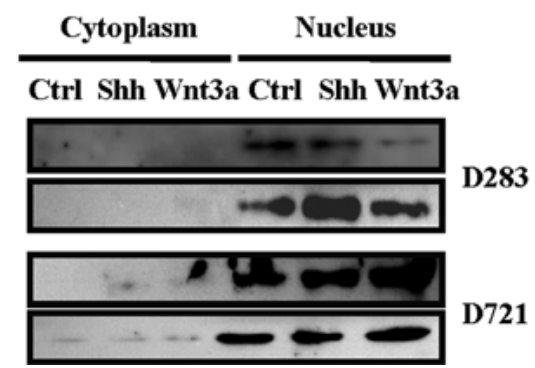

Figure 2. Heparanase pre-treatment modulates Shh/Wnt3a-dependent protein expression and subcellular distribution in MB cells. MB cell lines (D283 and D721) were pre-treated without (-) or with (+) active rhHPSE (200 ng/ml), then treated with Shh and/or Wnt3 (100 $\mathrm{ng} / \mathrm{ml}$ each), and the cytoplasmic/nuclear fractions $(60 \mathrm{mg}$ ) were subsequently analyzed by Western blotting. (A) GEF-H1, (B) $\beta$-catenin, (C) N-Myc and (D) fibrillarin were used as control specific for nuclear fractions.

these MB cells under serum-containing conditions by Western blotting. High GEF-H1/SDC4 protein expression was found in poorly invasive D283 compared to highly aggressive D721 cells. Moreover, inverse SDC1/SDC4 profiling was detected according to the MB cell invasive phenotype (Fig. 1D).

Heparanase modulates GEF-H1, $\beta$-catenin and $N$-Myc subcellular distribution in medulloblastoma. Recent evidence demonstrated that GEF-H1 functions to facilitate RhoA activity in the cytoplasm at sites of focal adhesion turnover, such as in regions of membrane ruffling (32). Therefore, GEF-H1 localization may influence cell migration and cytoskeletal dynamics. To characterize endogenous GEF-H1 expression and subcellular distribution in response to treatment with Shh or Wnt3a, and subsequent to pre-treatment with exogenous rhHPSE, nuclear and cytoplasmic fractions were isolated. GEF-H1 antibody detected both phosphorylated and non-phosphorylated forms of GEF-H1 in these subcellular compartments (Fig. 2A). Exposure to rhHPSE altered the GEF-H1 expression and its subcellular redistribution. The MB cell lines possessed not only a differential GEF-H1 expression and subcellular localization by rhHPSE, but also dependency upon Shh or Wnt3a treatment (Fig. 2A).

Since the expression and subcellular distribution of $\beta$-catenin is a crucial component of adherens junctions and co-transcription in Wnt signaling (33), signal transduction cross-talk between Shh and Wnt3a may be involved in MB, as demonstrated in other systems (34). Exposure to HPSE altered $\beta$-catenin expression and subcellular localization; differential $\beta$-catenin protein expression and subcellular redistribution were detected following Shh or Wnt3a treatment (Fig. 2B). D283 cells displayed primarily nuclear $\beta$-catenin, while
D721 cells exhibited robust cytoplasmic $\beta$-catenin content, increasing in response to $\mathrm{Shh} / \mathrm{Wnt} 3 \mathrm{a}$ (Fig. 2B). To note, the $\beta$-catenin content of D721 cells was drastically reduced subsequent to pre-treatment with active HPSE (Fig. 2B).

Since a burst of N-Myc expression has been associated with proliferation of external granular layer neurons of the developing cerebellum and MB (1), human MB model system was analyzed for N-Myc expression. Treatment with exogenous active HPSE may modulate the MB cell response to Shh or Wnt3a treatments. Cytoplasmic and nuclear fractions were probed for N-Myc protein expression, and N-Myc modulation by Shh or Wnt3a was distinctly observed (Fig. 2C). No N-Myc expression was detected in D283. Only exposure with Shh or Wnt3a induced N-Myc in the D721 cells. To note, HPSE abrogated N-Myc expression in the MB cell lines (Fig. 2C). The purity of the nuclear fractions was confirmed by probing with fibrillarin (Fig. 2D).

Heparanase modulates Gli gene expression in medulloblastoma. Regulation of Gli transcription factor genes is a key downstream read-out of activation of the Shh signal transduction pathway (1). Since Gli genes are activating (Gli1) or repressing (Gli2/Gli3) (35), the expression of the Gli genes subsequent to HPSE exposure was analyzed, followed by treatment with Shh or Wnt3a. Gli from the D283 cells were expressed under control conditions and HSPE treatment reduced Gli2/3 gene expression (Fig. 3). Treatment of D283 cells with Shh also resulted in Gli2/3 gene expression (Fig. 3). However, subsequent to treatment with exogenous HPSE, as without growth factor treatment, Gli2/3 gene expression was reduced (Fig. 3). The Gli gene expression from the poorly invasive D283 cells was modulated by treatment with 


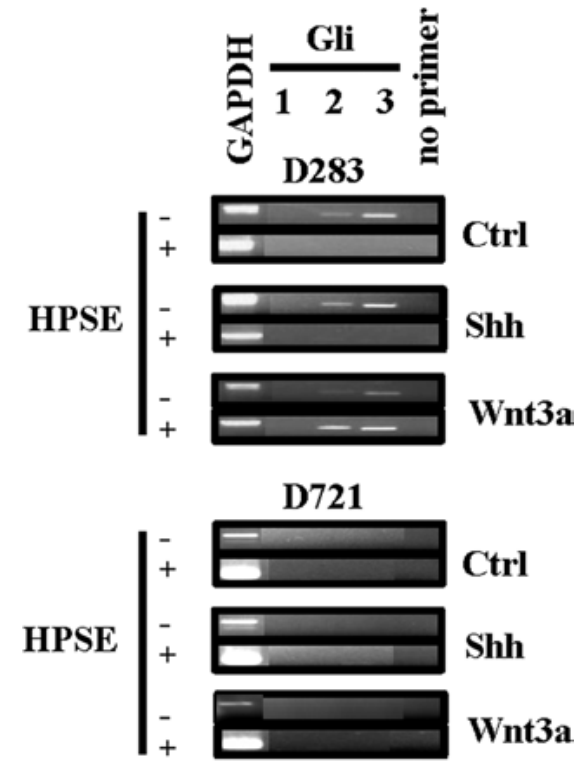

Figure 3. Heparanase (HPSE) pre-treatment modulates Shh/Wnt3adependent expression of Gli genes in human MB cells. Cells (D283 and D721) were pre-treated without (-) or with (+) active rhHPSE (200 $\mathrm{ng} / \mathrm{ml})$, and subsequently treated with Shh and/or Wnt3a (100 ng/ml each), RNA was isolated, and semi-quantitative RT-PCR was performed (Ctrl, not growth factor-treated). GAPDH was used as a positive control. Mastermix without the primer pair was used as a negative control and a test for contamination. PCR product sizes: GAPDH, 611 bp; Gli1, 572 bp; Gli2, 492 bp; Gli3, 416 bp.

exogenous HPSE, while not from the highly invasive D721 cells. To note, signal transduction cross-talk was demonstrated from the D283 cells, as Wnt3a treatment distinctly resulted in Gli2/3 gene expression (Fig. 3) (34). The Gli gene expression from the D721 cell line was quiescent in response to Shh or Wnt3a treatment, an indication that this signal transduction pathway is likely not involved in the highly invasive phenotype of these cells (Fig. 3).

Heparanase modulates Racl and RhoA activities in medulloblastoma. Cell motility requires a dynamic cytoskeleton $(36,37)$, and small GTPase Racl and RhoA activities are necessary in cytoskeletal dynamics $(36,37)$. To reveal the mechanisms of cell motility and determine whether heparanase modulates GTPase activity in medulloblastoma cells, Rac1 and RhoA activities in MB cells were measured following heparanase treatment. In D283 cells, heparanase pre-treatment significantly decreased Racl activity, while concomitantly increasing RhoA activity (Fig. 4). In these cells, HPSE facilitated the RhoA activity response induced by Shh or Wnt3a (Fig. 4B). Conversely, the D721 MB cell line displayed increased Rac1 activity as a general response to HPSE (Fig. 4A). HPSE pre-treatment resulted in increased Rac1 and RhoA activities in the D721 cells (Fig. 4). RhoA activity from the D721 cells decreased following Shh or Wnt3a treatment subsequent to HPSE pre-treatment, as opposed to the D283 RhoA activity response which was HPSE-facilitated (Fig. 4B). HPSE pre-treatment modified the Rac1/RhoA activity response to Shh and Wnt3a under every condition tested. To note, RhoA activity was increased subsequent to rhHPSE (Fig. 4B).
Heparanase modulates Shh and Wnt-induced medulloblastoma cell invasion. It is known that the invasive phenotype requires a dynamic cytoskeleton (38). Considering that cytoskeletal dynamics rely upon the activity of Racl and RhoA small GTPases (38), and that HPSE regulates Rac1 and RhoA activities, MB cells of varying invasive characteristics were pre-exposed (4) with HPSE, followed by Shh or Wnt3a treatment.

HPSE pre-treatment significantly increased the invasiveness of the $\mathrm{D} 283 \mathrm{MB}$ cells $(\mathrm{P}<0.05, \mathrm{P}<0.01)$ in the conditions tested (Fig. 5). Conversely, highly invasive D721 cells experienced less invasiveness in response to HPSE. Treatment with Shh or Wnt3a subsequent to HPSE did not restore D721 cell invasiveness (Fig. 5). Taken together, these data indicate the differential roles of HPSE in regulating MB cell invasion in highly invasive vs. non-invasive cell lines.

Heparanase modulates Shh and Wnt-induced medulloblastoma cell proliferation. $\mathrm{MB}$ is marked by a dramatic burst of cell proliferation from developing external granular layer neurons as a response to secreted growth factors from Purkinje neurons (1). Accordingly, the role of HPSE modulating MB cell proliferation was explored. No Shh/Wnt-induced proliferative responses to these treatments were noted in rapidly proliferative D283 cells. However, D721 cell proliferation was augmented in response to treatment with Shh (140\%) or Wnt3a $(156 \%, \mathrm{P}<0.05)$ (Fig. 6). Notably, the Shh/Wnt-induced proliferative response was not present subsequent to HPSE treatment (Fig. 6), indicating that HPSE and Shh/Wnt work in synergy to reduce cell proliferation in D721 cells.

\section{Discussion}

Interest concerning the roles of the HSPGs in MB is growing and has become a subject of continued investigations. In this study, we characterized the relevance of the HPSE/HSPG axis on Shh and Wnt3a signaling in a human MB cell system consisting of two lines with distinct tumorigenicities. We provide first-time evidence demonstrating i) a differential cell surface HSPG expression, notably of SDC1 and 4; ii) the identification of a guanine nucleotide exchange factor, GEF-H1, associated with SDC CT domain; iii) the importance of GEF-H1 in HSPG-modulated Shh and Wnt3a signaling affecting Rac1/RhoA activities and cell invasiveness; iv) the fact that active HPSE significantly affects Shh and Wnt3a signaling and alters the expression and localization of key downstream effectors, namely $\beta$-catenin and the Gli family of transcription factors.

Cell surface SDC1 and 4 are central in cell invasion and focal adhesion, and small GTPase activity is critical to cell motility $(11,13)$. We provided evidence that a small GTPase regulatory protein, GEF-H1, is differentially expressed under normal (serum-containing) growth conditions among $\mathrm{MB}$ lines previously characterized in regards to HPSE functionality towards invasiveness $(4,25)$. Recently, Sanz-Moreno et al indicated that the interchangeable plasticity of Rac1 vs. RhoA activity is important in tumor cell migration (38). GEF-H1 seems to be uniquely situated to participate in such interplay, since it binds and regulates both Racl and RhoA $(39,40)$, and thus can be considered a key regulator of tumor cell migration 
A

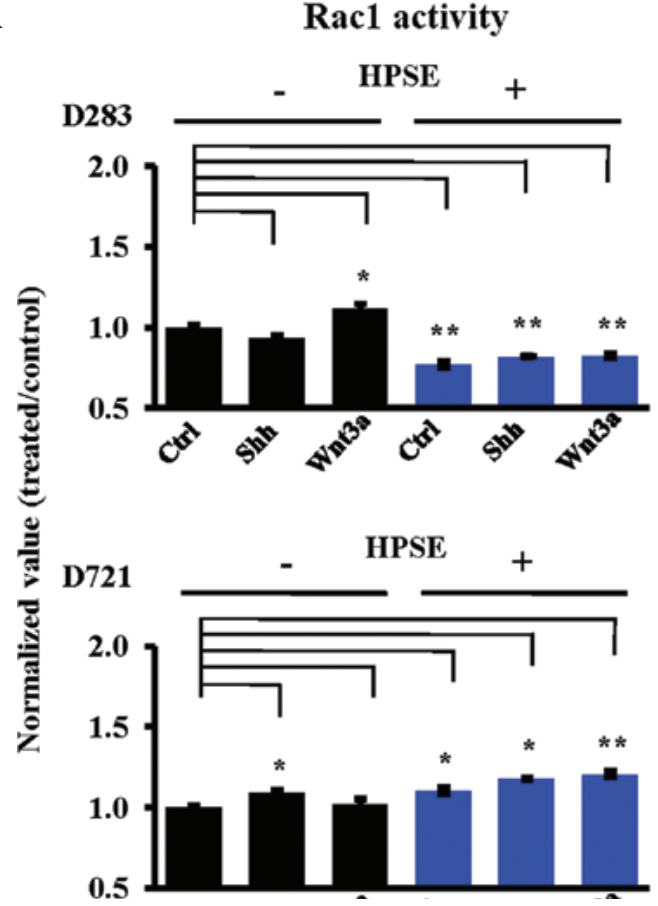

B

RhoA activity
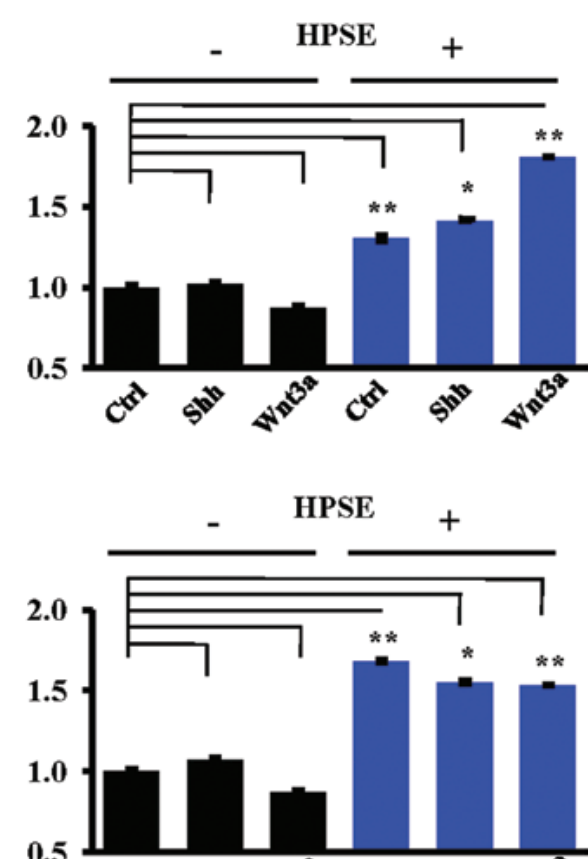

Figure 4. Heparanase pre-treatment modulates Shh/Wnt3a-dependent Rac1 and RhoA small GTPase activity in MB cells. Cells (D283 and D721) were pre-treated without (black bars) or with (blue bars) active rhHPSE (200 ng/ml), and subsequently treated with Shh and/or Wnt3a (100 ng/ml each). GTPase activities were subsequently measured $\left({ }^{*} \mathrm{P}<0.05 ;{ }^{* *} \mathrm{P}<0.01\right)$. (A) Rac1 activity assay $(\mathrm{n}=4)$. (B) RhoA activity assay $(\mathrm{n}=3)$.

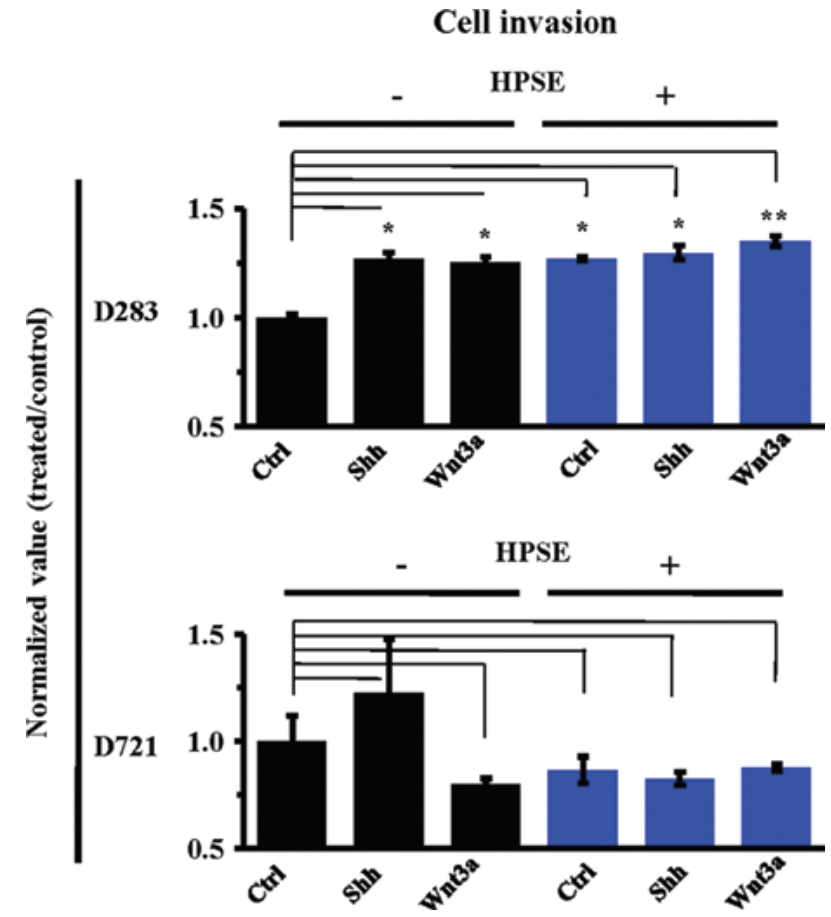

Figure 5. Heparanase pre-treatment modulates Shh/Wnt3a-dependent MB cell invasion. Cells (D283 and D721) were pre-exposed without (black bars) or with (blue bars) active rhHPSE $(200 \mathrm{ng} / \mathrm{ml})$, and subsequently treated with Shh and/or Wnt3a (100 ng/ml each). Invasion assays were subsequently performed, and the number of cells that invaded through the Matrigel-coated filters was quantified and statistically analyzed $\left({ }^{*} \mathrm{P}<0.05 ;{ }^{* *} \mathrm{P}<0.01 ; \mathrm{n}=4\right)$.

and phenotypic plasticity. This is of relevance as it refutes the expectation that a global RhoA preference throughout a cell is due to the presence of more GEF-H1 protein.

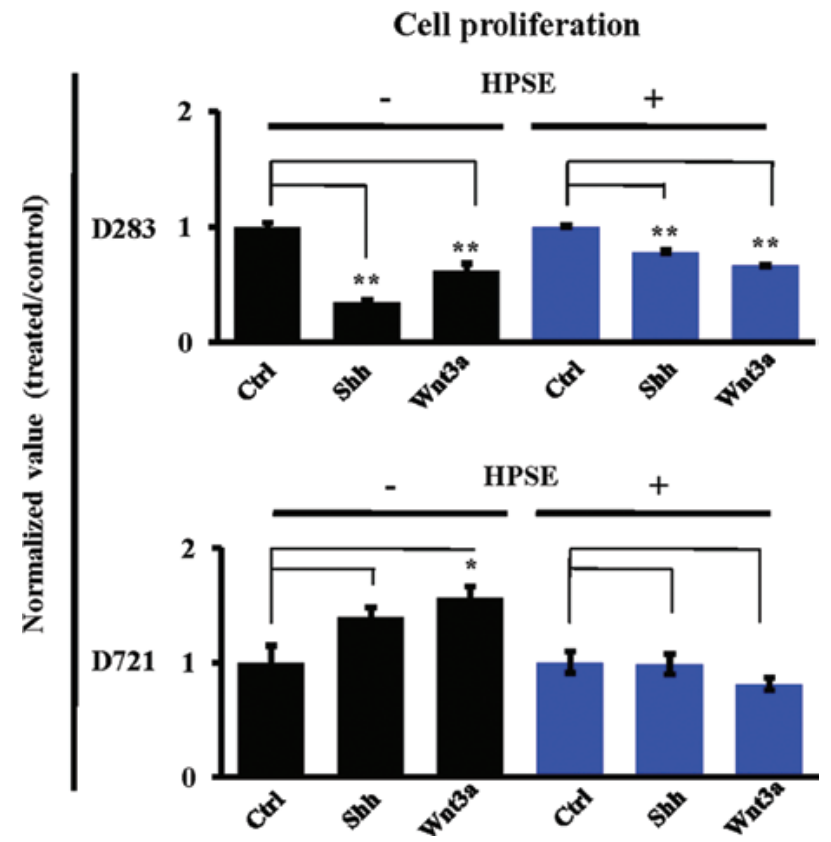

Figure 6. Heparanase pre-treatment modulates Shh/Wnt3a-dependent MB cell proliferation. Cells (D283 and D721) were pre-treated without (black bars) or with (blue bars) active rhHPSE (200 ng/ml), and subsequently treated with Shh and/or Wnt3a (100 ng/ml each). Cells were counted before and after HPSE pre-treatments to ensure cell number fidelity $\left(\mathrm{n}=4 ;{ }^{*} \mathrm{P}<0.05\right.$; ${ }^{* * *} \mathrm{P}<0.01$ ).

We addressed this issue by comparing RhoA activities between the D283 and D721 cells. We observed a correlation between HPSE-induced GEF-H1 expression (Fig. 2A) and decreased Rac1, but increased RhoA activity in the D283 
cells, a situation one would anticipate in association with GEF-H1 modulation of Racl and RhoA activities. We identified increased Rac1 and RhoA activities in HPSE-pre-treated D721 cells decreasing GEF-H1 expression in response to such treatment (Fig. 2A). This indicates that D721 may rely upon an alternate (or additional) Rho GEF (Fig. 5B) (32).

Similarly, and considering that the $\beta$-catenin status in MB tumors is clinically relevant (20), we observed primarily nuclear $\beta$-catenin in our least invasive D283 cells. By contrast, the more aggressive D721 cells had increased cytoplasmic localization of $\beta$-catenin augmented without the presence of heparanase. Furthermore, the Shh/Wnt treatment increased cytoplasmic $\beta$-catenin, and exposure to active HPSE abrogated $\beta$-catenin expression and localization in these cells (Fig. 2B).

Highly proliferative D283 cells did not express nuclear N-Myc constitutively or in response to growth factor stimulation (Fig. 2C). The less proliferative D721 cells proved to be the more growth factor-inducible and this finding correlated well with N-Myc expression (Fig. 2C).

Stimulation by Shh and/or Wnt has been demonstrated in other systems to induce Gli gene expression (41). The expression pattern of the three Gli genes has been termed the 'Gli code' as the resulting phenotypic outcomes of active or repressive can be predicated by the combination of Gli gene expression (35). We demonstrated that the less invasive D283 cells were Gli repressive under conditions without exogenous active HPSE. Highly invasive D721 cells were Gli inert, regardless of HPSE treatment. Furthermore, we showed that when a MB cell responds to Shh or Wnt3a by Gli gene expression, subsequent exposure to exogenous active HPSE will likely result in repressing Gli gene expression. Our data also indicated that D283 MB cells demonstrate Wnt/Gli crosstalk (Fig. 4). HPSE pre-treatment inhibited Rac1 activity, but facilitated RhoA activity in poorly invasive D283 cells. Thus, the ratios of Racl and RhoA activity in these cells can be modulated to favor RhoA GTPase activity, in response to exogenous active HPSE, which represses the Rac1 activity while concomitantly increasing GEF-H1 protein expression. This is particularly valid when GEF-H1 is highly present in the cytoplasm (Fig. 2A). To note, the greatest increase in Rac1 GTPase activity was in the D721 cells, and this response was Shh-dependent. Although it has been shown that Shh down-regulates MB cell growth when cells are explanted into culture (42), our study is still relevant to gain insights into the cellular physiology of MB. For example, we previously demonstrated modulation of GEF-H1 induced signaling by HPSE in brain metastatic settings (43). However, it remains to be demonstrated whether or not GEF-H1 expression is consistently associated with decreased invasiveness in other tissue types. HPSE pre-treatment augmented Rac1/RhoA small GTPase activity, which is indicative of increased cytoskeletal dynamics.

Furthermore, we demonstrated that HPSE pre-treatment increased the invasiveness in a RhoA-dependent manner under conditions of increased GEF-H1 expression which augmented the invasiveness of D283. Conversely, we found that the highly invasive D721 cells did not show reduced Rac1 activity to accompany the increased RhoA activity in response to HPSE pre-treatment and decreased GEF-H1 expression while increasing RhoA activity. Therefore, an alternate Rho
GEF may facilitate the increased RhoA activity in these cells. Along with reduced GEF-H1 expression associated with HPSE pre-treatment, D721 also demonstrated reduced invasiveness associated with increased RhoA activity.

In summary, we demonstrated a correlation between cell surface HSPG-induced mechanisms and MB cell invasiveness linking SDCs to GEF-H1 and Rac1/RhoA activities, the involvement in Shh/Wnt signaling pathways and their modulation by the presence of exogenous active heparanase. We also demonstrated roles for heparanase in regulating $\mathrm{MB}$ cell proliferation and adhesion in addition to its established heparan sulfate chain degrading activity. These findings provide impetus in further deciphering the HPSE/HSPG axis to affect gene regulation in MB and to generate synergies towards the discovery of novel markers for MB clinical intervention.

\section{Acknowledgements}

We thank Dr Israel Vlodavsky and Dr Neta Ilan (The Bruce Rappaport Medical Faculty, Technion, Israel) for kindly providing the rhHPSE. We also thank Dr Richard Cook, Director of the BCM mass spectrometry core facility for MALDI-TOF MS/MS analyses, and Dr Anita Chandler (Department of Molecular Physiology and Biophysics at BCM) for the expert editorial assistance. This study was supported by NIH grant 2R0-1 CA 086832 (to D. Marchetti).

\section{References}

1. Guessous F, Li Y and Abounader R: Signaling pathways in medulloblastoma. J Cell Physiol 217: 577-583, 2008.

2. Flora A, Klisch TJ, Schuster G and Zoghbi HY: Deletion of Atoh1 disrupts Sonic Hedgehog signaling in the developing cerebellum and prevents medulloblastoma. Science 326: 1424-1427, 2009.

3. Ribi K, Relly C, Landolt MA, Alber FD, Boltshauser E and Grotzer MA: Outcome of medulloblastoma in children: long-term complications and quality of life. Neuropediatrics 36: 357-365, 2005.

4. Marchetti D, Mrak RE, Paulsen DD and Sinnappah-Kang ND: Neurotrophin receptors and heparanase: a functional axis in human medulloblastoma invasion. J Exp Clin Cancer Res 26: 5-23, 2007.

5. Gottardo NG and Gajjar A: Current therapy for medulloblastoma. Curr Treat Options Neurol 8: 319-334, 2006.

6. Yauch RL, Dijkgraaf GJ, Alicke B, et al: Smoothened mutation confers resistance to a hedgehog pathway inhibitor in medulloblastoma. Science 326: 572-574, 2009.

7. Vaillant C and Monard D: SHH pathway and cerebellar development. Cerebellum 8: 291-301, 2009.

8. Cool SM and Nurcombe V: Heparan sulfate regulation of progenitor cell fate. J Cell Biochem 99: 1040-1051, 2006.

9. Rubin JB, Choi Y and Segal RA: Cerebellar proteoglycans regulate sonic hedgehog responses during development. Development 129: 2223-2232, 2002.

10. Mythreye K and Blobe GC: Proteoglycan signaling co-receptors: roles in cell adhesion, migration and invasion. Cell Signal 21: 1548-1558, 2009

11. O'Connell MP, Fiori JL, Kershner EK, et al: Heparan sulfate proteoglycan modulation of Wnt5A signal transduction in metastatic melanoma cells. J Biol Chem 284: 28704-28712, 2009.

12. Iozzo RV: Heparan sulfate proteoglycans: intricate molecules with intriguing functions. J Clin Invest 108: 165-167, 2001.

13. Sanderson RD and Yang Y: Syndecan-1: a dynamic regulator of the myeloma microenvironment. Clin Exp Metastasis 25: 149-159, 2008.

14. Beauvais DM and Rapraeger AC: Syndecans in tumor cell adhesion and signaling. Reprod Biol Endocrinol 2: 1-12, 2004. 
15. Tkachenko E, Rhodes JM and Simons M: Syndecans: new kids on the signaling block. Circ Res 96: 488-500, 2005.

16. Fux L, Ilan N, Sanderson RD and Vlodavsky I: Heparanase: busy at the cell surface. Trends Biochem Sci 34: 511-519, 2009.

17. Ilan N, Elkin $M$ and Vlodavsky I: Regulation, function and clinical significance of heparanase in cancer metastasis and angiogenesis. Int J Biochem Cell Biol 38: 2018-2039, 2006.

18. Goodrich LV, Milenkovic L, Higgins KM and Scott MP: Altered neural cell fates and medulloblastoma in mouse patched mutants. Science 277: 1109-1113, 1997.

19. Berman DM, Karhadkar SS, Hallahan AR, et al: Medulloblastoma growth inhibition by hedgehog pathway blockade. Science 297 $1559-1561,2002$

20. Fattet S, Haberler C, Legoix P, et al: Beta-catenin status in paediatric medulloblastomas: correlation of immunohistochemical expression with mutational status, genetic profiles, and clinical characteristics. J Pathol 218: 86-94, 2009.

21. Symons M and Segall JE: Rac and Rho driving tumor invasion: who's at the wheel? Genome Biol 10: 1-4, 2009.

22. Sanz-Moreno V and Marshall CJ: Rho-GTPase signaling drives melanoma cell plasticity. Cell Cycle 8: 1484-1487, 2009.

23. Ilina $\mathrm{O}$ and Friedl P: Mechanisms of collective cell migration at a glance. J Cell Sci 122: 3203-3208, 2009.

24. Avalos AM, Valdivia AD, Munoz N, et al: Neuronal Thy-1 induces astrocyte adhesion by engaging syndecan- 4 in a cooperative interaction with alphavbeta3 integrin that activates PKCalpha and RhoA. J Cell Sci 122: 3462-3471, 2009.

25. Sinnappah-Kang ND, Kaiser AJ, Blust BE, Mrak RE and Marchetti D: Heparanase, TrkC and p75NTR: their functional involvement in human medulloblastoma cell invasion. Int J Oncol 27: 617-626, 2005.

26. Birkenfeld J, Nalbant P, Yoon SH and Bokoch GM: Cellular functions of GEF-H1, a microtubule-regulated Rho-GEF: is altered GEF-H1 activity a crucial determinant of disease pathogenesis? Trends Cell Biol 18: 210-219, 2008.

27. Nardella C, Lahm A, Pallaoro M, Brunetti M, Vannini A and Steinkuhler C: Mechanism of activation of human heparanase investigated by protein engineering. Biochemistry 43: 1862-1873, 2004.

28. Ridgway LD, Kim EY and Dryer SE: MAGI-1 interacts with Slol channel proteins and suppresses Slol expression on the cell surface. Am J Physiol Cell Physiol 297: C55-C65, 2009.

29. Xian X, Gopal S and Couchman JR: Syndecans as receptors and organizers of the extracellular matrix. Cell Tissue Res 339: 31-46, 2009.

30. Alexander CM, Reichsman F, Hinkes MT, et al: Syndecan-1 is required for Wnt-1-induced mammary tumorigenesis in mice. Nat Genet 25: 329-332, 2000
31. Dovas A, Yoneda A and Couchman JR: PKCbeta-dependent activation of RhoA by syndecan-4 during focal adhesion formation. J Cell Sci 119: 2837-2846, 2006.

32. Nalbant P, Chang YC, Birkenfeld J, Chang ZF and Bokoch GM: Guanine nucleotide exchange factor-H1 regulates cell migration via localized activation of RhoA at the leading edge. Mol Biol Cell 20: 4070-4082, 2009.

33. Hartsock A and Nelson WJ: Adherens and tight junctions: structure, function and connections to the actin cytoskeleton. Biochim Biophys Acta 1778: 660-669, 2008.

34. Alvarez-Medina R, Cayuso J, Okubo T, Takada S and Marti E: Wnt canonical pathway restricts graded Shh/Gli patterning activity through the regulation of Gli3 expression. Development 135: 237-247, 2008.

35. Ruiz i Altaba A, Mas C and Stecca B: The Gli code: an information nexus regulating cell fate, stemness and cancer. Trends Cell Biol 17: 438-447, 2007.

36. Ridley AJ and Hall A: The small GTP-binding protein rho regulates the assembly of focal adhesions and actin stress fibers in response to growth factors. Cell 70: 389-399, 1992.

37. Ridley AJ, Paterson HF, Johnston CL, Diekmann D and Hall A The small GTP-binding protein rac regulates growth factorinduced membrane ruffling. Cell 70: 401-410, 1992.

38. Sanz-Moreno V, Gadea G, Ahn J, et al: Rac activation and inactivation control plasticity of tumor cell movement. Cell 135 510-523, 2008

39. Ren Y, Li R, Zheng Y and Busch H: Cloning and characterization of GEF-H1, a microtubule-associated guanine nucleotide exchange factor for Rac and Rho GTPases. J Biol Chem 273: 34954-34960, 1998.

40. Birkenfeld J, Nalbant P, Bohl BP, Pertz O, Hahn KM and Bokoch GM: GEF-H1 modulates localized RhoA activation during cytokinesis under the control of mitotic kinases. Dev Cell 12: 699-712, 2007

41. Ulloa F and Marti E: Wnt won the war: antagonistic role of Wnt over Shh controls dorso-ventral patterning of the vertebrate neural tube. Dev Dyn 239: 69-76, 2009.

42. Sasai K, Romer JT, Lee Y, et al: Shh pathway activity is downregulated in cultured medulloblastoma cells: implications for preclinical studies. Cancer Res 66: 4215-4222, 2006.

43. Ridgway LD, Wetzel MD and Marchetti D: Modulation of GEF-H1 induced signaling by heparanase in brain metastatic melanoma cells. J Cell Biochem: Aug, 2010 (E-pub ahead of print). 